\title{
La Reforma del Estado en las Provincias Argentinas: Una visión desde sus condicionantes estructurales*
}

\author{
Roxana Rubins ${ }^{1}$ \\ Horacio $\mathrm{Cao}^{2}$
}

\section{Resumen}

El propósito de este trabajo es estudiar los condicionantes estructurales de la reforma del Estado en las provincias argentinas. Los resultados revelan que a) los desequilibrios regionales de la República Argentina fueron modelados por los diferentes hitos que jalonan su historia. El estado que emerge de este proceso es el que pone de manifiesto la hegemonía de una región (la pampeana, situada en el centro-oeste) sobre el resto del país; b) la ocupación del espacio argentino está entonces caracterizada por una subordinación diferenciada hacia esta región; algunos segmentos se incorporan como proveedores de productos agrícolas para el mercado interno, otros se vinculan directamente con el mercado mundial, sentando las bases para una inmigración posterior y otros se acoplan como periferias no funcionales desde el punto de vista productivo, aunque aportan estabilidad política al sistema de dominación y se transtorman en reservorios de abundante y no caliticada mano de obra; c) a partir de 1930 se hace más complejo un sistema que ya había establecido sus bases varias décadas antes. Las políticas keynesianas desarrolladas por esa época, en el marco de un federalismo basado en el acuerdo entre regiones, generan una maraña de subsidios y exenciones que permitió hacer florecer a la región pampeana y mantener una cierta dinámica a las regiones periféricas. Como subproducto de esta situación se genera una suerte de especialización, en donde las provincias construyen sistemas y aparatos políticos de dominación que se entroncan con las tradiciones

\section{Recibido: 19-11-97. Aceptado: 12-3-98}

Investigadora del Centro de Estudios de Políticas Administración y Sociedad (CEPAS), de la Asociación de Administradores Gubernamentales de Argentina. Investigador del CEPAS, Administrador Gubernamental. 
clientelares de América Latina, mientras que el Gobierno Federal exhibe un sistema de dominación relativamente "neutro" e "impersonal". Se concluye que esta situación da una cierta especificidad al modo de funcionamiento de las Administraciones Provinciales, lo que deben tomarse en cuenta a la hora de definir su nuevo perfil, que estará directamente marcado por los cambios que, a partir de los ' 90 ', genera la deconstrucción del modelo keynesiano.

Palabras clave: Reforma del Estado, Argentina, Administraciones provinciales, ocupación del espacio, Gobierno Federal.

\section{State Reform in Argentinian Provinces: A Vision of Structural Impositions}

\section{Abstract}

The purpose of this paper is to study the structural impositions of state reform in Argentinian provinces. The results reveal that : a) the regional disequilibriums of the Argentinian Republic were modeled by the different period which mark its history. The state which emerged from this process is one that demonstrates the hegemony of one region (the Pampas, situated in the northwest) over the rest of the country; $b$ ) the occupation of the argentinian territory is characterized by a differentiated subordination towards this region, some segments were incorporated as providers of agricultural produce for internal markets, others directly oriented to world markets, which provided the basis for later immigrations, and other regions were peripheral and nonfunctional from a productive point of view, even though they were importante form the point of view of political stability and domination, and they became resevoirs of abundant yet non-qualified labor; c) beginning in 1930, the system which had been established over several decades became more complex. The Keynesian economic policies developed in this period in the framework of a federalism based on interregional agreements generated an entanglement of subsidies and exonerations which permitted the pampas region to flower and to maintain a certain dynamic over the other peripheral regions. As a sub-product of this situation, a sort of specialization was generated in which the provinces constructed systems and political aparatus of domination which connected them with traditional latinamerican clients, while the Federal government demonstrated a system of relative neutrality or impersonality. As a conclusion this situation gives certain specificity to the manner of functioning of provicial administrations, which should be taken into account at the moment of defining new profiles, which are direcly marked by the changes which, beginning in the 90 s, were generated by the deconstruction of the Keynesian Model.

Key words: State reform, Argentina, Provincial Administration, Spacial occupation, Federal government 


\section{Introducción}

El proposito de este trabajo es estudiar las condicionantes estructurales de la reforma del Estado en las Provincia Argentinas. El trabajo pretende resumir diferentes "papers" que a partir de 1993 se elaboraron alrededor de la temática de la Reforma del Estado en el ámbito provin$\mathrm{cial}^{3}$ de la República Argentina. Se trata de un trabajo, que presenta los principales avances logrados previamente, pero a su vez sintetiza, de alguna manera, las diferentes perspectivas.

En este sentido, se trabajó sobre la base de exponer las principales conclusiones a las que se arribó en los siguientes temas:

- Cómo se ocupó el espacio en la Argentina: su origen $y$ evolución.

- Una descripción esquemática del modelo de dominación en las provincias.

- La crisis económica y el ajuste: impacto sobre las economías regionales.

- El rol dinamizador de la reforma del Estado y sus condicionantes

Debemos destacar que las conclusiones preliminares de nuestra investigación resultaron lo suficientemente estimulantes como para iniciar dos líneas de trabajo posteriores, tanto sobre aspectos organizacionales del funcionamiento del aparato del Estado, como sobre el rol a cumplir por el Estado en los procesos de desarrollo regional ${ }^{4}$.

\section{El esquema de ocupación del espacio en la Argentina}

Cuando se analizan los desequilibrios regionales, tanto en la faz productiva, institucional, cultural como política de las provincias argentinas, suele olvidarse que éstos fueron modelados por el transcurso íntegro de la historia de nuestro país, remitiéndose la mayoría de los analistas al período en que la heterogeneidad estructural que caracteriza a los espacios regionales adoptó su forma más reciente: la etapa de mercado internista posterior a 1930.

Aquí interesa resaltar que la construcción de la Nación Argentina es fruto de un proceso de diferenciación regional, que se plasmó en un Estado construido con base en la hegemonía de una de las regiones que la integran, la pampeana (Para observar ubicación de las regiones en la República Argentina ver mapa en el Gráfico No. 1). Esta región impuso, no sin violencia, un modelo político, económico y social, que sólo pudo evolucionar manteniendo una unidad - a veces precaria- a

3 Se denominan provincias a las 23 jurisdicciones estaduales que, con alto nivel de autonomia conforman el segundo nivel de estado de la República Argentina. En nuestro caso, como ocurre en casi todas las investigaciones sobre el tema, se le agrega a estas 23 jurisdicciones la ciudad de Buenos Aires, Capital Federal del país, que tiene un status juridico particular de "Cludad Autónoma".

4 Sobre estas lineas de trabajo se basan los proyectos de tesis doctoral (Universidad de Buenos Aires) que los autores están realizando. 
Grafico No. 1

Mapa de Argentina con Identificación de Provincias y Regiones

\section{Ne Provincia}

1 Jujuy

2 Salta

3 Catamarca

4 Tucumán

5 Santiago del Estero

6 Formosa

7 Chaco

8 Misiones

9 Corrientes

10 Entre Rios

11 La Rioja

12 San Juan

13 Mendoza

14 San Luis

15 Córdoba

16 Santa $\mathrm{Fe}$

17 Buenos Aires

18 Ciudad de Buenos Aires (1)

\section{Región de Cuyo}

Noreste Argentino

NEA

Argentino

NOA
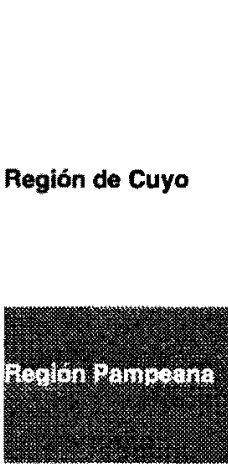

19 La Pampa

20 Neuquén

21 Río Negro

22 Chubut

23 Santa Cruz

24 Tierra del Fuego (2)

(1) Ciudad Autónoma

(2) El nombre completo es el de "Provincia de Tierra del Fuego, Antärtida Argentina e Islas del Atlántico Sur". Comprende, entre otras, las Islas Malvinas, Sandwichs del Sur, Georgias del Sur, Orcadas del Sur, Shetland del Sur y el territorio Antártico Ar. gentino

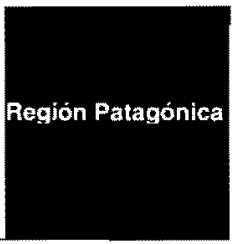


partir de generar espacios de integración y de exclusión con el sector hegemónico.

La afirmación presentada utiliza como esquema analítico los modelos centro - periferia (Amín, 1983 - Blomstrom y Bjom, 1990 y Evers, 1989), los que, en los trabajos elaborados aparecen modificados para evitar los errores que, según entendemos, contienen ${ }^{5}$ con relación al rol de los diferentes agentes sociales y de las instituciones. Asimismo, se incorporan algunas caracterizaciones que apelan al lenguaje de los modelos del dualismo económico, pero evitando suponer la falta de relación entre los sectores "modernos" y no modernos de la economia, a efectos de incorporarlos plenamente en el esquema analítico.

De este modo, nuestra "imagen" de ocupación de los espacios regionales parte de un proceso que se origina en la etapa colonial, con una división "espacial" del trabajo que brinda un marco estructural a partir del cual se construirán, a la par, el Estado y la nación Argentina a lo largo del siglo $\mathrm{XIX}$.

En la etapa colonial, el asentamiento español en la Argentina se organiza desde el emprendimiento minero del Potosi. Este esquema de ocupación del territorio genera espacios funcionales y disfuncionales a este centro hegemónico, básicamente alrededor de la disponibili- dad de mano de obra indígena para incorporarse a la actividad expansiva del período. Así, la región pampeana posee importancia solamente como región fronteriza, a partir de la cual facilitar o impedir el contrabando.

La docilidad, abundancia, o capacidad para la resistencia de los indigenas de cada sub-espacio regional sobredeterminan el modo en que cada región se incorpora al emprendimiento exitoso del momento, existiendo incluso autores que explican los clásicos problemas fronterizos con Brasil del período colonial a partir de la disponibilidad de indios en las Misiones Jesuíticas (Rock, 1994)

La falta de centralidad de la región pampeana con respecto al centro hegemónico explicaria, en parte, su éxito posterior: el ganado transformado en cerril, ocupando un espacio sin competidores ecológicos, y multiplicándose inmensamente, presta el punto de partida para la "oligarquía con olor a bosta" (Halperín Donghi, 1980) que construye la Nación a partir del siglo XIX. También esta multiplicación a lo Garcia Márquez justifica el esquema de tenencia de la tierra que presentan las pampas, asi como los vínculos particulares que quienes desarrollan este modelo de acumulación establecen con el ascendente imperialismo inglés desde el siglo XVII en adelante (Rock 1994) ${ }^{6}$.

Por ejemplo, transformar en homogéneamente "explotados" a los diferentes sectores sociales que desarrollan su actividad en un espacio regional particular, $\sin$ atender a las diferencias sociales entre ellos, sin considerar las contraprestaciones del centro hacia las periferias.

En el libro citado, se afirma lo siguiente: "Todo el comercio estaba en manos de los portugueses, que no estaban interesados en exportar productos ganaderos... sino la plata que llegaba de Potosí por Córdoba (pag.58)". Esto habría producido continuos problemas políticos en la Buenos Aires colonial, que sólo se habrían resuelto a partir de la alianza entre Gran Bretaña y Portugal a partir de comienzos del siglo XVIII, incorporando los cueros al intercambio. 
La construcción de la hegemonía portuaria y pampeana ocupa casi todo el siglo XIX, a lo largo del cual se libra una guerra civil que sella la estructura regional argentina. Entre los intentos de resistencia posteriores a la Revolución de Mayo (1810), hasta los alzamientos aislados que se verifican durante la presidencia de Sarmiento (1868/1874), se impone y logra la hegemonía de un modelo de acumulación basado en la integración a partir de la agricultura y la ganadería de clima templado al mercado mundial. Este modelo de acumulación es impuesto generando ganadores y perdedores de esta modalidad de vinculación con el exterior, no solo sociales, sino también políticos $y$ regionales.

La fase de equilibrio que cierra en 1930 , está caracterizada por una subordinación diferenciada al modelo de ocupación del espacio en el que algunas regiones $^{7}$ se insertan e incorporan como proveedoras de productos agrícolas para el mercado interno como es el caso de Cuyo, y parte del NOA y el NEA (ver Grafico No. 1).

Algunas regiones se vinculan directamente con el mercado mundial, sentando las bases para una nueva colonización (el caso emblemático es el de la Patagonia). Otras, forman parte de una periferia no funcional desde el punto de vista productivo, pero sí político: aportan estabilidad política al sistema de dominación: Es el caso de provincias de menor desarrollo relativo que comienzan a desarrollar una especialización política de "sostenes del sistema" que aún puede reconocerse en su rol en la nación.
Diferentes autores resaltan el rol del Estado Nacional en la "difusión" de este esquema de dominación (Halperín Donghi, 1980; Rock, 1994; Oszlack, $1984,1985,1989)$, que mediante instrumentos de cooptación (ferrocarriles, juzgados, escuelas, inversión pública, etc.) y de represión (fuerte presencia del ejército y la policía, intervenciones federales, etc.) consolida un equilibrio regional que resistirá hasta la crisis de 1930 para reformularse.

El siguiente período abarca hasta comienzos de la década del ' 70 , y está signado por la inestabilidad institucional y politica que caracteriza a todas las burguesias desarrollistas latinoamericanas en su incorporación a la nueva división internacional del trabajo que reconoce como centro hegemónico a Estados Unidos.

La construcción de un esquema de acumulación mercado - internista, con fuerte protección respecto del mercado mundial, y con una amplia difusión de la legislación social que favorece la incorporación al mercado nacional de una vasta gama de sectores sociales, permite la gestación de una nueva etapa de equilibrio regional, que no disuelve la heterogeneidad estructural que caracteriza a nuestra sociedad, aunque le permite adquirir otro perfil.

La región hegemónica es ahora el asiento fundamental de los principales emprendimientos industriales, alimentados por mano de obra proveniente de las diferentes regiones del país, que abastecen de materias primas industriales y de alimentos a la región central. Una amplia 
red de subsidios cruzados y omisiones de cobro permite generar un país más integrado, con mejores indicadores sociales, y una amplia cobertura estatal para los diferentes actores sociales y regionales, los que sin embargo no adquieren una base suficiente como para emprender proyectos que permitan cerrar la brecha con las regiones más avanzadas.

La utilización al máximo de los mecanismos de renta de monopolio a través de diferentes regulaciones estatales ${ }^{8}$ permite la supervivencia de las capas sociales más atrasadas, evitando los procesos de expropiación que se pondrian en marcha de someterlos a una mayor competencia capitalista, y aportando apoyo político para la supervivencia de las expresiones políticas más conservadoras de las distintas provincias.

Las áreas menos pobladas (la $\mathrm{Pa}$ tagonia), sujetas a un fuerte proceso de colonización, adquieren un muy superior dinamismo a partir de nuevos emprendimientos extractivos (petróleo, gas, carbón) y agrícolas (el alto valle del Río $\mathrm{Ne}$ gro) que el Estado sostiene mediante la inversión pública o distintas medidas de promoción.

En resumen, el equilibrio geográfico logrado entre las distintas zonas del pais para la etapa del Estado del Bienestartenia, esquemáticamente, las siguientes tipologías regionales:

- Área central: Posicionada sobre la Región Pampeana, permitía la obtención de las divisas externas necesarias para mantener en funcionamiento el es- quema de sustitución de importaciones, a la vez que tenía un importante peso en el desarrollo del mercado interno. Fruto de corrientes migratorias y de la reproducción de su situación hegemónica frente al resto del país, fue generando un desequilibrio abrumador en cuanto a tamaño y dinamismo frente a la globalidad del resto del pais.

- Periferias Prósperas: Aquellas que con la expansión del mercado interno tuvieron la posibilidad de generar productos para la zona "central" $y$, sobre la base de la expansión del ingreso, registrar ritmos de crecimiento económico apreciables logrando, por lo menos, crear empleos suficientes como para retener el crecimiento vegetativo de su población (Ferrer, 1980). Forman parte de este núcleo las provincias que contienen los tradicionales complejos agrícolas y agroindustriales extrapampeanos (vitivinícola en Cuyo; azúcar en el NOA, frutícola en núcleos Patagónicos, algodón en el NEA, etc.)

* Periferias Rezagadas: Regiones que quedaron fuera de las corrientes más dinámicas del sistema de relaciones asimétricas. Son aquellas que no desarrollaron o desarrollaron muy tenuemente actividades de "exportación" significativas hacia la zona "central". De este modo, estas provincias deterioraron su participación en la distribución de la población del pais y registran los menores índices de actividad económica y niveles de vida. Estas regiones no estuvieron directamente vinculadas a la reproducción del

8 Para un análisis detallados de estos mecanismos ver Rubins/Cao (1996). De todas formas se volverá sobre el tema más adelante 
modelo hegemónico; así el impacto que el mismo tuvo sobre ellas, fue mínimo. Forman parte de esta categoría las provincias de Catamarca, La Rioja, Jujuy, Santiago del Estero (NOA), Formosa (NEA).

*Periferias Despobladas: Por último encontramos regiones en las que, a lo largo del período analizado, se realizan tareas de "ocupación" que garantizan la soberanía y la creación de la infraestructura necesaria para su efectiva integración (ejemplificadas por las Provincias Patagónicas, sin potencialidad de articulación dependiente con la potencia hegemónica anterior).

Debe hacerse notar que en muchos casos las provincias, si bien pueden ser incluidas en una tipología hegemónica, la misma puede no ser única. Así por ejemplo Santa $\mathrm{Fe}$ (Area Central) tiene emprendimientos de periferias prósperas (el algodón del norte), y zonas rezagadas / despobladas (el noroeste, lindante con Santiago del Estero).

El esquema de equilibrio regional que se desarrolla en este período toca su fin con el golpe militar de 1976, momento a partir del cual se ingresa en un período de crisis que comienza a resolverse recién a partir de los ' 90 , cuando la hiperinflación arrasa con todas las expectativas de refuncionalizar el esquema anterior. Así como la crisis del país hace difícil encontrar un diagnóstico único, del mismo modo se torna complejo establecer parámetros de clasificación que reemplacen esta diferenciación ya obviamente superada.

\section{El esquema de dominación}

El esquema de dominación nacional es relativamente diferente del que se verifica en el ámbito local. Con fínes analíticos, supondremos que en el ámbito nacional se cumplen las pautas particulares que permiten suponer un divorcio relativo entre Estado - Sociedad Política y Clase Dominante - Sociedad Civil, que sustenta los niveles de autonomía relativa del Estado, necesarios para legitimar las instituciones del capitalismo. Esto se afirmará aunque se tiene conciencia de que en la mayoría de las situaciones concretas de la actividad política y/o empresaria, tal autonomía relativa no puede afirmarse sin más pruebas, sin dejar de mencionar la "complicación analítica" que supone incorporar en el esquema, el rol de correa de transmisión que cumplen los sectores dominantes a escala nacional respecto de la inserción general del espacio mercantil que denominamos "Nación Argentina" con relación al mercado mundial.

En principio, tres de los principales supuestos de existencia del Estado capitalista no se cumplen en forma acabada a nivel local. Existe además algún tipo de correlación que profundiza esta característica cuanto más acentuada sea su condición de periferia:

i) La equiparación de los individuos en el ámbito económico como propietarios privados libres que exige en el ámbito político su reconocimiento como ciudadanos también formalmente libres e iguales. En el ejercicio del poder social estos principios formales imponen una forma de 
dominación aparentemente neutra, impersonal y desinteresada de toda diferencia material. La utilización clientelar del estado y las formas tradicionales de dominación que son típicas de los Estados Provinciales de las regiones periféricas enturbian la posibilidad de los individuos de presentarse en el ámbito económico como propietarios privados y libres. Por este motivo, tampoco las formas de dominación se presentan como neutras e impersonales.

ii) El Estado aparece sostenido por cargas a la comunidad, emergiendo por sobre ella, articulando los intereses generales por encima de la competencia en el mercado. En el caso de los Estados Provinciales, el Sector Público es sostenido por transferencias nacionales, por lo que se pondera la capacidad de negociación con el estado nacional por sobre la capacidad recaudatoria local. En lo económi$c o$, buena parte de las actividades provinciales descansa básicamente en un esquema sostenido por el nivel de gasto del Sector Público Provincial. Este sector es en casi todas las provincias, el principal empleador ${ }^{9}$, el que genera la demanda para que subsista el sector comercial, el principal (casi único) inversor en infraestructura, el que permite el desarrollo de una red de servicios, etc.

iii) Por último, en el modelo clásico se sostiene que la administración del Estado queda a cargo de personas especlficas - políticos profesionales y un aparato burocrático subordinado a ellos- que aparecen como meros instrumentos para medir y expresar el (ilusorio) interés general. En las sociedades duales, tales como las de las provincias periféricas, en los sectores de menor desarrollo relativo la Administración Pública Provincial, se encuentra bajo la égida directa de grupos personalizados (más aún, es normal encontrar casos de nepotismo)

Los fondos con que el Estado $\mathrm{Na}$ cional soporta a las provincias, sirven a las estructuras clientelares típicas de los Estados Provinciales para reproducir el sistema, con lo cual cumplen con su función de velar por las condiciones generales de reproducción y de imponer tales condiciones en contra de la resistencia que puedan encontrar en individuos o grupos, hasta el punto que el aparato del estado aparece cooptado directamente por familias o clanes. Por otro lado, la debilidad de la burguesía provincial frente al Estado y las limitaciones de toda la estructura, hacen que no exista competencia en el sentido capitalista del término asi como tampoco existen sectores sociales que sustenten ( $y$ exijan) otros comportamientos de los partidos políticos $y$ parlamentos.

Una explicación tentativa para estos comportamientos en el ámbito local, puede encontrarse en la extrema simpleza de las sociedades y economias de la mayoría de las provincias, y en las formas de dominación paternalistas. Tornan sobreabundantes los canales clásicos de las democracias liberales en donde se "parlamentan" complejos intereses, y en los cuales la competencia entre las fracciones de la clase dominante resuelve, por el consenso y la negociación, el "interés general". 
Los comportamientos clientelares que se registran en la mayoría de los aparatos de estado locales no deben buscarse en la existencia de desvíos patológicos esporádicos. Aparecen a partir de la situación de retraso general, y de la función que, ahora o en otra etapa, tuvo/tiene el aparato estatal en la organización del mercado nacional.

Este retraso y/o simpleza no es tomado en cuenta por las instituciones provinciales que, más allá de diferencias anecdóticas, tienen estructuras muy similares, basadas en normas pensadas para estados capaces de tener un desarrollo autónomo.

En resumen, el cuadro en el que podemos encajar los esquemas de dominación regionales puede presentarse, tentativamente, del siguiente modo:

a) El Estado Provincial que formalmente es autónomo del Estado Nacional, está en los hechos subordinado a él. Esta subordinación deviene de la incapacidad financiera de cada provincia en particular, la que a su vez es un subproducto de su retraso relativo respecto de la región hegemónica.

b) La sociedad civil, entretanto, presenta características también particulares y diferentes de las de la región central. La economía de cada provincia particular aparece hegemonizada por uno o más emprendimientos locales ("economías regionales") que permiten vincular a la clase dominante local con el mercado nacional. Sin importar si el emprendimiento es actualmente funcional al ordenamiento territorial giobal, sitúa a los terratenientes/productores que se especializan en él como la fracción principal de la clase dominante local. Las clases medias aparecen, entonces, o bien ligadas al/a los emprendimientos locales o a la gestión del aparato del estado o a las fracciones urbanas proveedoras de los servicios mínimos imprescindibles para su reproducción. En tanto la producción tradicional esté en crisis, todos ellos dependen fuertemente de las transferencias nacionales para su supervivencia.

c) En el marco de la extrema rudimentariedad de la organización económica, y bajo las caracteristicas históricas de una dominación de tipo patrimonialista, no se encuentran los actores sociales capaces de generar un escenario que posibilite un rol independiente para el poder legislativo o para la justicia. Tendencialmente, en la actividad política real, estos poderes son cooptados por clanes o grupos económicos o familiares.

Dijimos anteriormente que esta particular articulación entre Estado y Sociedad Civil obedecía al retraso relativo que presentaba la estructura económica de algunas provincias. En el próximo punto, se profundizará este análisis.

\section{La estructura económica y el rol del Estado}

La estructura económica que presenta actualmente el país es un reflejo de ciertos equilibrios regionales en tren de modificación.

Así, entendemos que el Area Central actúa como locomotora del resto del país, siendo asiento principal de las actividades que vinculan al país con el resto del mundo. Esta región presenta los emprendimientos de mayor dinamismo, concentra casi el $70 \%$ de la población total y más del $75 \%$ del Producto Bruto Geográ- 
fico en un área que ronda el $20 \%$ del total del territorio nacional (Secretaria de Asistencia para la Reforma Económica Provincial, 1995) ${ }^{10}$.

En las regiones periféricas, se asientan emprendimientos funcionales y no funcionales al Área Central. Hasta mediados de los 70 , podian reconocerse una variedad de "economías regionales" que atravesaba a la mayoría de las provincias que aqui hemos denominado periferias prósperas: la producción de algodón, azúcar, yerba mate, ganado ovino, vitivinicultura, manzana, pera, productos de granja (aves, huevos, etc.), tabaco, citricos, etc., eran producidos para el mercado interno (con una gran concentración de consumidores en el Área Central) por las provincias de Cuyo, NEA, NOA y, en menor medida, la Patagonia. Las periferias no funcionales aparecían con una gran estabilidad en su población total, merced a la continua emigración de población hacia las regiones más prósperas, $\sin$ haber podido desarrollar un emprendimiento que les permitiera insertarse de mejor modo en la economía nacional. En el desempeño de cada una de estas actividades (incluyendo las de la región central) aparece como esencial el rol cumplido por el Estado a partir de la década del ' 30 , profundizándose su actividad desde la posguerra.

El mapa productivo argentino reconoce el impacto de un conjunto de medidas desarrolladas alrededor de diferentes instrumentos de política económica, aplicadas sobre la totalidad del entramado productivo nacional que entrarán en crisis sólo a partir de mediados de los '70. Entre ellas podemos mencionar:

- Un formidable complejo de medidas destinadas a la protección del mercado interno (aranceles, cupos de importación, medidas para-arancelarias, prohibiciones directas de incorporar mercancias del exterior, etc.). Diferentes autores calculan un arancel promedio de $143 \%$ en el momento de auge del esquema de protección a fines de los ' 60. (Ramos, 1993; Lucangeli, 1989)

- Un sinnúmero de subsidios explicitos o implícitos para favorecer el desarrollo de determinadas industrias: tarifas subsidiadas de diferentes empresas públicas de: luz, gas, energia, transporte o teléfonos; préstamos bancarios a tasa subsidiada por debajo de la inflación; devoluciones de impuestos, aranceles o derechos; deducciones varias de impuestos; desmantelamiento de algunas actividades estatales competitivas (el ejemplo es la supresión de los tranvías para favorecer el desarrollo de la industria automotriz).

- Un esquema de reproducción de la fuerza de trabajo destinado a sostener el mercado interno (implantación del sistema de obras sociales, sistema jubilatorio y de seguridad social, leyes de negociación colectiva, de asociaciones sindicales y patronales). 
En paralelo con estas medidas, pero destinados a proveer de insumos locales y alimentos para el mercado interno, aparecen otros instrumentos de politica económica que promueven la actividad de las "economías regionales": créditos subsidiados por mecanismos de oferta (líneas de redescuento estacionales del Banco Central, encajes diferenciados), organismos reguladores de la actividad regional (Junta Nacional de Granos, Junta Nacional de Carnes, Dirección Nacional del Azúcar, Ente Regulador de la Yerba Mate, Instituto Nacional de Vitivinicultura), inversión pública nacional en infraestructura, subsidios en la energía o los combustibles, la instalación de empresas nacionales en lugares remotos del país, etc. (Domeniconi et al. 1992; Squiglia y Delgado 1993).

Esta intensa actividad estatal favoreció un proceso de desarrollo económico que tuvo como resultados más inmediatos el fortalecimiento de un núcleo industrial sumamente concentrado en la región pampeana, la retención de población en las provincias periféricas (población vinculada a los emprendimientos dinámicos regionales), un intenso proceso de poblamiento en las provincias despobladas, y el estancamiento en las más rezagadas. Ninguno de los instrumentos aplicados resolvió las desigualdades regionales, ni proveyó los elementos mínimos para lograr procesos más equilibrados de desarrollo.

A mediados de los ' 70 , con la crisis del petróleo importada y la crisis política local mediante, la totalidad del esquema ingresa en un período crítico, fundamentalmente debido a la imposibilidad de continuar financiando su reproducción. Las fuentes que financiaron el modelo anterior estaban prácticamente agotadas (Torre y Gerchunoff, 1988). El sistema jubilatorio, montado en los ' 40 y expoliado desde los ' 60 debía comenzar a pagar; la producción agropecuaria, cuya renta era capturada a través de diferentes mecanismos, se estancó ${ }^{11}$.

Además el país era, en ese entonces, un fuerte importador de petróleo $y$, por ende, de inflación externa; los esquemas de redistribución del ingreso generados en la etapa del peronismo, pero que conocieron su auge durante el gobierno de Ongania y permitieron apuntalar el mercado interno llegaban a una etapa en la que requerian no solo redistribuir el crecimiento, sino también la riqueza. De este modo, la vigencia del esquema se continuó a crédito: esta es la base del fenomenal proceso de endeudamiento externo que experimentó el país sobre fines de la década de los ' 70 , permitiendo prolongar la agonía del modelo mercadointernista.

La nacionalización de la deuda externa a partir de 1982 perfeccionó los mecanismos de imposición de una reestructuración productiva que ya no podía financiarse localmente. La crisis económica permanente que atravesó la década del ' 80 funcionó como disciplinador político para imponer la reorientación de la totalidad de la economia para su reinserción en el mercado mundial. Pero sólo la administración justicialista pudo -fundaaftosa. 
mentalmente después de dos crisis hiperinflacionarias - iniciar una profunda reforma del estado que facilitara esa reinserción.

A partir de 1990, se suceden la batería de leyes y proyectos de reordenamiento de la economía y el Estado que han remodelado la estructura del pais: Las leyes de Reforma del Estado y privatizaciones permiten la venta o concesión de las principales empresas públicas (teléfonos, gas, electricidad, petróleo, carbón, obras sanitarias, siderurgia, bancos nacionales), las que se complementan con otras para la concesión de rutas, empresas de energía nuclear, o desmantelamiento y cierre de empresas deficitarias (Empresa Líneas Maritimas Argentinas, Instituto Nacional de Reaseguros, etc.).

En otro plano, la Nación transfiere a las provincias la mayoría de los servicios sociales que hasta el momento prestaba directamente (hospitales, escuelas, comedores, hoteles, etc.), e impulsa a través de diferentes "Pactos fiscales" la privatización y reordenamiento de los entes públicos provinciales.

Los Decretos de desregulación $\mathbf{N}^{\circ}$ $2.284 / 91,815 / 92,1.493 / 92,1.187 / 93$, etc. proveen un nuevo ordenamiento que favorecerá el desarrollo de nuevas actividades en el ámbito provincial (tales como los referentes a minería) y la cancelación de otros que hasta el momento se creían viables.
Dentro de este nuevo ordenamiento no tienen vigencia los entes reguladores estatales, que tenían como objetivo garantizar precios sostén, fijar cupos a la producción de diferentes productos regionales, adelantar crédito subsidiado a los pequeños productores, etc., de forma tal de garantizar un piso de renta para los pequeños propietarios ${ }^{12}$.

El impacto sobre las economías regionales es mayúsculo, por la doble situación de eliminación de los entes reguladores y la deconstrucción de la red jurídica que regulaba la estructura productiva. Se estima que todas estas medidas impactarán fuertemente sobre los pequeños productores de las periferias prósperas, terminando con una estructura de tenencia de la tierra en la cual la expropiación de estos segmentos no estaba en juego.

A la reestructuración de la producción local se le suma:

a) El ajuste y las privatizaciones a nivel Nacional, que dejan una importante secuela de despidos (con fuerte impacto en las provincias petroleras, con explotación siderúrgico con regimientos militares importantes).

b) Las privatizaciones en que incurren las provincias, que también acarrean fuertes despidos y otros efectos sobre la actividad productiva local ${ }^{13}$.

c) El auge de nuevos emprendimientos de estilo "enclave", como el desarrollo de proyectos mineros en distintas

12 Y suculentas ganancias para los grandes propietarios

13 La privatización de los bancos provinciales termina con líneas de financiamiento muy particulares, que fueron posibles merced a un manejo clientelar del crédito y de las entidades bancarias oficiales en las provincias. Si bien el manejo clientelar del crédito no garantiza el acceso de todos los productores al mismo, si permite a una buena parte (la clientela) persistir como pro- 
provincias, pesqueros en el litoral Atlántico, o de pasta de papel en el NEA.

Todas estas situaciones hacen suponer que el escenario en que habrán de desenvolverse los hechos políticos y económicos del próximo período está en un profundo tren de modificación.

\section{El rol dinamizador de la Reforma del Estado y sus condicionantes}

Los procesos de Reforma del Estado que han encarado las provincias, parten de una caracterización del funcionamiento de los aparatos estatales que puede resumirse como sigue:

" Compartimentalización de la Organización: el estado aparece como escindido, sin coordinación ni comunicación, lo que hace imposible optimizar el uso de recursos y circuitos.

* Ineficiencia e ineficacia de los circuitos: Como un rasgo general, los diferentes circuitos (de información, de autoridad, de decisión, etc.) presentan serias dificultades para su funcionamiento. Los mismos no tienen canales objetivos, sino que se desarrollan en virtud de instancias informales 0 ajenas a la problemática en sí (poder e influencia, seguimiento personalizado, etc.).

" Falta de Información: No se tie- ne información, o la que se tiene no se encuentra adecuadamente presentada, o no se encuentra en tiempo y forma en el lugar apropiado. 1) Para la alimentación de circuitos: lo que promueve la ya detaliada ineficiencia en general de los circuitos. 2) Para la toma de decisiones: lo que hace que las decisiones se tomen insertas en marcos de incertidumbre.

* Deficiencias en la infraestructura, mobiliario y útiles y herramientas de trabajo: El trabajo cotidiano se realiza en lugares inadecuados, muchas veces sobrepoblados de personal, sin los mínimos elementos necesarios para el desarrollo de tareas.

- Problemas Normativos: Los problemas normativos generan que las acciones del Estado deban seguir un farragoso camino de control interno que garantice su legalidad, y aún así, es común su anulación posterior por vicios normativos. Básicamente los mismos pueden dividirse según tres características principales: 1) Las deficiencias de las normas complejas, que presentan problemas técnicos o fueron copiadas para regular temas en medios muy distintos al provincial. 2) No existen sistematizaciones (o no se puede acceder a ellas) que permitan un rápido y fácil acceso a las normativas buscadas, 3) El complejo normativo no conforma un Sistema, sino que es un conjunto desarticulado de normas, con fuertes niveles de redundancia y lagunas jurídicas.

* Falta de políticas hacia el Empleo Público que incrementen su pro-

ductores en períodos de crisis. El cambio, a partir de la privatización, es que los créditos se tornarán "exigibles" (aspecto desusado del crédito en el ámbito provincial), y accederán al mismo aquellos que puedan garantizar su repago (otro aspecto inusual del negocio financiero en las provincias). 
ductividad: Esta baja productividad puede atribuirse básicamente a la falta de capacitación, originada en las deficiencias de los mecanismos de reclutamiento, $y$ agravada por la inexistencia de una política de formación de los agentes del estado. Por otro lado también debe hacerse notar la falta de incentivos, ya que la carrera administrativa solamente existe en el papel, y se tiene por sabido que así como el reclutamiento fue hecho en términos clientelares, la "carrera" en el estado se llevará adelante en términos de cercanía con el poder y no en virtud del trabajo realizado.

El resultado de esta estructura es una administración sumisa, que se organiza sobre una base dual: un canal legalista, de un obsesivo ritualismo burocrático, el que obviamente nunca logra resultados y que está reservado para el público que no puede o no sabe hacer uso del jerarquizado sistema de favores que rige en los sistemas clientelares. Un canal jerarquizado, informal, ad hoc, utilizado por los grupos que ejercen el poder y por el que se despliegan las tramitaciones que se consideran importantes.

Sin embargo se considera que este diagnóstico no agota la complejidad de la situación por la que atraviesan los estados provinciales. Para completar el panorama, es preciso acotar la eficacia supuesta para las reformas administrativas, ya que el núcleo del problema radica en la particular interacción entre Estado y Sociedad Civil que puede verificarse en las sociedades regionales.
Por este motivo, si se comprende el esquema de funcionamiento de los aparatos estatales como una "caja negra", en cuyos "bordes" se concreta la vinculación entre Sociedad Civil y Sociedad Política, a partir de un ordenamiento normativo que, como dijimos anteriormente, no refleja exhaustivamente el real funcionamiento de ese tipo de sociedades ${ }^{14}$, es que se piensa que las iniciativas que no profundicen en la reformulación de estos vínculos están destinadas a un continuo "volver a empezar".

En particular, es notable la dinámica interna del nivel político, el que en el marco del aparato del Estado puede dividirse según el rol funcional que ocupa:

- El Ministerlo de Relaciones Políticas encargado de la relación con el Poder Legislativo y con los actores sociales relevantes (CGT, Iglesia, Partido Gobernante, Partidos Opositores, etc.), generalmente posicionado en el Ministerio de Gobierno, en la Secretaría General de la Gobernación o un poco en ambos.

- Los Ministerios de Gestión, en los que se agrupan las unidades encargadas de llevar adelante los sistemas de educación, salud y acción social. Estas tres incumbencias ejecutan más del $50 \%$ del presupuesto, teniendo un amplio despliegue en toda la provincia y una posición principalísima dentro de las tareas que realiza el estado provincial. Los ministerios de "ges- 
tión" tienen a su cargo el gerenciamiento de sistemas complejos, que se articulan con actores sociales de alta activación política y poder (especialmente sindicatos en el caso de educación, a los que se le suman federaciones de clínicas, colegios médicos, laboratorios, etc. en el caso salud).

- El Ministerio del Ajuste, encargado de conseguir los recursos y asignar los fondos. Este Ministerio está posicionado sobre la estructura hacendaria de la provincia. En los casos de crisis el ministro del ajuste reduce sus tareas a dos tipos de acciones: conseguir fondos para el pago de sueldos, para lo que pasa la mayoría de su tiempo tramitando los mismos ante el estado federal; $y$ "sentarse sobre la caja", minimizando los gastos de operación y segando todos los nombramientos.

Las otras funciones de los estados provinciales ocupan un lugar secundario dentro de la agenda politica, social y organizacional. Por ejemplo las unidades encargadas de regular $y / 0$ promover la producción, que generalmente tienen muchas oficinas aplicadas al seguimiento de cada área en particular (Secretarías de industria, agricultura y/o minería, Direcciones de control y/o desarrollo de la producción, entes de seguimiento de emprendimientos específicos, etc.) producen un impacto casi nulo, fuera de su función en la estructura generadora de empleo público y de burocracia pasible de estar incursa en acciones de corrupción.
Presentando la situación en términos polares, que en los hechos tienden a trocarse en situaciones intermedias, puede decirse que a cada tipo de Ministerio le corresponde un tipo de cuadro de dirección. En los Ministerios de Gestión y de Relaciones Políticas se encontrará una mayoría de cuadros que denominaremos "Políticos" y en los Ministerios de Ajuste, de cuadros que denominaremos "Técnicos".

Los cuadros técnicos son el vehículo por el cual el Ministerio de Ajuste se relaciona con las estructuras del ajuste nacional, y a su vez, la forma en que los organismos que monitorean la marcha de la economia nacional (el FMI) extienden su influencia hasta las provincias.

Por su parte, los cuadros políticos tienen una posición caricaturescamente enfrentada a la de los cuadros técnicos. Su supervivencia y crecimiento dependen de la capacidad de distribuir prebendas de forma tal de mantener el sector que lo apoya y, de ser posible, extender10.

Mientras los técnicos son los vehiculos de la reformulación del Estado en los términos que se establecen desde el mercado mundial, los políticos son los que buscan que el accionar del Estado permita, por la vía clientelar, la reproducción de las fracciones sociales y productivas más expuestas. Ambas soluciones presentan sus propios límites: por la crisis de financiación (los políticos) o por explosión social o presión de los grupos de poder locales (los técnicos).

En este escenario de conducción, las unidades orgánicas se desenvuelven en forma autónoma, repitiendo ritualmente las tareas que en algún momento fue- 
ron aprendidas, recibiendo la atención de la conducción en el momento en que surgen problemas en el área o que deben entregar algún producto que pueda generar algún tipo de acumulación para la conducción. En otros momentos, los cuadros de conducción ocupan su tiempo en neutralizarse mutuamente; esto es, defenderse, o atacar a la otra ala de gobierno.

Sobre la debilidad de este aparato de Estado se asientan las últimas transferencias ${ }^{15}$ realizadas por el Estado Nacional. Las mismas tienden a profundizar una especialización en gestión de funciones "sociales", quedando en manos del Estado Nacional acciones de transferencia de ingresos, que demandan principalmente una cierta racionalidad administrativa y que en general pueden considerarse de menor complejidad que las que deben llevar adelante las provincias. En esta misma acción de transferencia, las provincias reciben incumbencias que ya tienen desarrollado un modelo de gestión y que, bajo una cierta inercia, son difíciles de reformar hacia modelos que puedan adaptarse (en volumen y complejijdad) a la escala de la organización (el estado provincial) que las debe gerenciar.

Estas incumbencias tienden a presionar sobre las erogaciones, exigiendo a algunos aparatos exhaustos (en particular de las provincias rezagadas) un gran esfuerzo organizacional, requiriendo acciones vinculadas con la calidad de las prestaciones, la eficiencia, la gestión de recursos humanos y materiales, etc. (Lerner, 1992).

En un escenario cruzado por estas múltiples tensiones se han emprendido una pluralidad de acciones de reforma, con resultados muy diferenciados según las jurisdicciones. Demás está decir que las mismas han representado esfuerzos inmensos para los Estados Provinciales.

Las principales políticas que promovió la Nación en este sentido estuvieron dadas por los pactos fiscales, los fondos fiduciarios y los préstamos con condicionalidades. A partir de ellas el Estado Central indujo politicas de reforma con la contrapartida de ofrecer a las provincias ventajas financieras. A continuación se resumen algunas de las políticas de reforma más importantes:

a) Se han privatizado la mayoria de los bancos provinciales (segmento del sistema financiero denominado Banca Oficial de Provincia), lo que redefine una serie de mecanismos clientelares que facilitaron, en el pasado, la reproducción de las "economias provinciales" y los aparatos políticos del Estado.

b) Se han privatizado algunas empresas de energía y de obras sanitarias. Estos entes, incapaces en el pasado de cobrar por los senvicios prestados, fueron una fuente de problemas de financiamiento para los estados provinciales.

c) Se ha limitado el ingreso de nuevos agentes a las posibilidades de financiamiento del estado provincial en muchas provincias.

d) La casi totalidad de los estados provinciales se adhirió a la Ley Federal de Educación, que impone una reforma educativa de importantes dimensiones. 
e) Se ha buscado articular las políticas tributarias de las provincias con la de la nación. Los resultados obtenidos han sido parciales.

f) Se ha intentado coordinar las políticas de desregulación llevadas adelante por la nación con las provinciales. También en este rubro los resultados son parciales.

g) Diversos préstamos de organismos financieros internacionales ${ }^{16}$ se han desembolsado sujetos a la transferencia de tecnologías, o asistencia técnica para su ejecución. Esto redundó en reformas en los subsistemas de salud, educación o viales, tendientes a mejorar la eficacia administrativa de estos subsistemas.

Sin embargo, las probabilidades de éxito de estos programas aparecen condicionadas a lograr una reformulación que permita reinsertar a las economías y sociedades provinciales en el nuevo esquema espacial que intenta el pais en estos dias.

\section{Conclusión}

Toda crisis supone no sólo una situación confusa, en la que no se distinguen ganadores ni perdedores, sino una oportunidad para replantear el rol de cada actor social en el esquema que emerge.

Desde este punto de vista, la reformulación del rol del Estado tanto en el ámbito nacional como provincial no es el único motivo para la aparición de la crisis, aunque pese a ello, pueda constituirse en una herramienta que modifique y reordene completamente el escenario en que se desenvuelve.

Las acciones concretas de Reforma del Estado nacional (privatizaciones, transferencias de servicios sociales, redimensionamiento de los aparatos militares y burocráticos centrales) han impactado profundamente en los niveles de actividad provinciales. A este conjunto de medidas deben sumarse los impactos de los acuerdos fiscales, que han llevado a las clases dominantes locales a transferir herramientas clientelares de suma importancia, tales como los bancos provinciales, las empresas de energía o las cajas de jubilación. Esta redefinición del rol del Estado ha inducido una crisis profunda en los aparatos políticos tradicionales, generando una tensión importante entre diferentes estrategias a adoptar.

Los Estados provinciales se ven obligados a elegir entre dos fuerzas contrapuestas que impulsan a quienes conducen el aparato del Estado a actuar en pos de la siguiente dicotomía: para facilitar la reconversión, el Estado tiende a comprometer su esfuerzo en desarrollar acciones que permitan la rearticulación en la economía provincial con los emprendimientos más dinámicos en curso, imbuidos ahora por una lógica transnacional ${ }^{17}$; en tanto para mantener la gobernabilidad, debe desarrollar acciones que

16 Tal es el caso de los préstamos denominados "Provincias $\mid$ y $\| \mathbb{1}^{\text {; }}$ el Programa de Reforma Económica Provincial y el Programa de Desarrollo Provincial, entre otros.

17 Que para desarrollar emprendimientos modernos pugna por la extraterritorialidad y el menor contacto posible con las estructuras públicas locales, tendiendo a transformar a los emprendimientos modernos en "enclaves". 
permitan sostener la paz social, profundizando un perfil clientelar que atraviesa horizontalmente a la casi totalidad de las estructuras politicas de las provincias argentinas, pero habiendo perdido las herramientas principales para desenvolver estas políticas.

Por este motivo, es que se propuso al comienzo de este análisis que la crisis podía ser pensada desde un punto de vista que considere las oportunidades que abre, en lugar de las estructuras que sepulta en su desarrollo. La reformulación del rol del Estado, en la medida que permita una nueva articulación tanto económica, como política o social entre los actores que desarrollan su accionar en el ámbito provincial con el espacio nacional, facilita el nacimiento de un nuevo equilibrio interregional en el que es importante que quienes participan de ese proceso reconozcan las señales que muestran el fin de la etapa anterior, y la construcción de una nueva.

La imposibilidad de reconocer que la etapa anterior ha finalizado impedirá obtener una mejor posición relativa para cada espacio regional que intente reinsertarse, transformando todos los esfuerzos en pos del ajuste en preludios de nuevos ajustes, en una espiral descendente que sólo tenderá a profundizar más la crisis en desarrollo.

\section{Referencias bibliográficas}

Agosin, Manuel y Ffrench Davis, Aicardo (1993). "La liberalización comercial en América Latina" Revista de la CEPAL N ${ }^{2} 50$.

Arnin, Samir (1983) "El desarrollo desigual" Planeta - Agostini, Barcelona.
Blomström, Magnus y Björn, Hettne (1990) "La teoría del desarrollo en transición", F.C.E., México.

Domeniconi, Héctor; Gaudio, R; Guibert, Armando (1992) "La desregulación en Argentina. Los primeros pasos de su implementación". Boletín Informe Techint No 271, Argentina jul/set.

Esteso, Roberto y Capraro, Héctor (1989), “Algunos elementos para el análisis de las relaciones entre el estado federal y las provincias", Cuademos IIPAS $N^{2} 1$, Fundación Friedrich Ebert, Buenos Aires.

Evers, Tilman (1989) "El estado en la periferia capitalista", Siglo XXI Editores, Buenos Aires.

Ferrer, Aldo (1980). "La Economía Argentina, las Etapas de su desarrollo $y$ Problemas Actuales" Fondo de Cultura Económica, Buenos Aires.

Ferreyra, Hugo - Rodríguez, Angel - Sarabia, Leticia - Seijas, Marcelo - Tomasino, Roberto - (1994). Informe AG Transterencia de Servicios Educativos en Revista Aportes para el Estado y la Administración Gubemamental - Año 1 - № 2 - Buenos Aires, Octubre.

Halperín Donghi, Tulio (1980) "Proyecto y Construcción de una nación. Argentina 1946-1880" Biblioteca Ayacucho, Caracas.

Lemer, Emilia (1992). Cambios recientes en la relación fiscal nación - provincias - Facultad de Ciencias Económicas - UBA - Trabajo presentado en el IX Congreso Nacional de Administración Püblica - Mendoza.

Lucángeli, Jorge (1989) Política comercial y desempeño industrial. La experiencia argentina de los últimos 40 años Boletín Informe Techint $N^{2} 259$, Argentina set/oct. 
Oszlak, Oscar (1984) "Notas Criticas para una Teoría de la Burocracia Estatal", en "Teoría de la Burocracia Estatal" - Oscar Oszlak (Compilador) PAIDOS, Buenos Aires.

Oszlak, Oscar(1985). "La Formación del Estado Argentino" Editorial Belgrano $1^{2}$ REIMPRESION - Buenos Aires.

Oszlak, Oscar. (1989). "El Estado Nacional en el Interior Significación e Impactos" En Administración Pública y Socledad N 3, Publicación periódica del IIFAP, Córdoba - Argentina, Julio .

Ramos, Joseph (1993) "Crecimiento, crisis y viraje estratégico". Revista de la CEPAL $n^{\circ}$ 50, Agosto.

Rock, David (1994) "Argentina 1516-1987. Desde la colonización española hasta Raúl Alfonsin" Alianza Edito- rial, $4^{\circ}$ Edición Argentina, Buenos Aires.

Secretaria de Asistencia para la Reforma Económica Provincial (SAREP) (1995). "Datos para el Análisis del Sector Público" Ministerio del Interior, Buenos Aires, Julio.

Squiglia, Eduardo y Delgado, Ricardo (1993) "Desregulación y competitividad. Evaluación de la experiencia argentina". Boletín Informe Techint $\mathrm{N}^{\circ}$ 276, Argentina Oct/Dic.

Torre, Juan Pablo y Pablo Gerchunoff (1988) "El papel del Estado" - Revista IDEA, Argentina, Mayo.

Vaca, Ángel - Francés, Mónica - Cao, Horacio (1997) "Empleo Público en las Provincias Rezagadas". En: Revista Aportes para el Estado y la Administración Gubernamental - Año 4 - $N^{0} 8$. Buenos Aires. 